\title{
ВЛИЯНИЕ МЕХАНИЧЕСКОЙ ОБРАБОТКИ НА СТРУКТУРУ И СОРБЦИОННЫЕ СВОЙСТВА ТИТАН-ЦИРКОНИЙСОДЕРЖАЩЕГО СОРБЕНТА ПРИ СОРБЦИИ УРАНА ИЗ МОДЕЛИРУЮЩИХ СОСТАВ МОРСКОЙ ВОДЫ РАСТВОРОВ
}

\author{
(C) 2017 А. Н. Нуриев, М. А. Рагимли \\ Институт катализа и неорганической химии имени академика М.Нагиева \\ НАН Азербайджана, пр. Г. Джавида, 113,AZ-1143 Баку, Азербайджан \\ e-mail:chem@science.az \\ Поступила в редакцию 26.04.2017 г.
}

\begin{abstract}
Аннотация. Изучено влияние механической обработки сорбента Термоксид-5 с содержанием 6.2 вес.\% $\mathrm{ZrO}_{2}$ на сорбцию урана из моделирующих состав морской воды растворов. При использовании свежеизмельченных мелкодисперсных фракций обнаружен эффект неравновесной разупорядоченности кристаллической решетки сорбента в виде пиков на кинетических кривых, исчезающих по истечению определенного времени. Полученные результаты позволили рассчитать значения энергии активации и эффективные коэффициенты диффузии дефектов в Термоксиде-5 при различных температурах. Установлено, что появление характерных максимумов на кинетических кривых связано с резким увеличением скоростей сорбции вследствие перестройки структуры сорбента в момент фазового перехода. Полученный эффект подтвержден данными рентгенофазового анализа. Интерпретация полученных результатов привела к выводу, что интенсивное измельчение сорбентов дестабилизирует их структуру и резко увеличивает скорость сорбции, а при стабилизации происходит полное восстановление сорбционных свойств.
\end{abstract}

Ключевые слова: уран, диоксид титана, сорбент, разупорядоченность структуры, сорбция, рентгенофазовый анализ.

\section{ВВЕДЕНИЕ}

Известно, что среди неорганических сорбентов различной природы, широко применяемых для извлечения, концентрирования и разделения многих катионов, наиболее перспективным сорбентом по воспроизводимости ионообменных, кинетических и механических свойств признан диоксид титана $[1,2]$. Но при длительном хранении, как и многие неорганические сорбенты, $\mathrm{TiO}_{2}$ подвержен старению, т.е. потере своих первоначальных сорбционных качеств.

Еще в средине XX века японскими исследователями было замечено, что лучшими сорбционными характеристиками, чем чистая гидратированная двуокись титана, обладает гидратированная двуокись титана, полученная из ильменита, содержащая до нескольких процентов двуокиси циркония. На этой основе российскими исследователями разработан смешанный сорбент гидратированных двуокисей титана и циркония [3], известный под товарным знаком « Термоксид-5».
Превосходство сорбционных свойств сорбентов типа «Термоксид-5» объясняется разупорядоченностью их структур вследствие введения в ходе синтеза в $\mathrm{TiO}_{2}$ анатазной модификации неизоморфной примеси двуокиси циркония [4].

Установление эффекта разупорядоченности кристаллической структуры сорбента, способствующего появлению новых активных центров и улучшению его сорбционных характеристик, послужило руководством для синтеза ряда эффективных материалов путем внедрения в основу добавок оксидов других металлов с низкой константой растворимости $\left(K_{\mathrm{s}}=10^{-51}-10^{-54}\right)$ для извлечения ценных микроэлементов из водных растворов [57]. Другим направлением в активировании свойств $\mathrm{TiO}_{2}$ является обработка его с помощью электрофизических методов. Перспективным направлением для активации свойств $\mathrm{TiO}_{2}$ является воздействие на него постоянным электрическим полем [8].

В литературе имеются сведения о достижении разупорядоченности структуры гидроксида тита- 
на под влиянием ультразвука [2]. Разупорядоченности кристаллической структуры решетки можно достигнуть, как известно [9], и с помощью механической обработки (измельчения) сорбента. Такая обработка ведет не только к изменению гранулометрического состава сорбента, но и приводит к образованию больших количеств нарушений структуры (разупорядоченность структуры), что оказывает существенное влияние на его сорбционные характеристики.

В литературе отсутствуют сведения о влиянии механической обработки на разупорядоченность структуры и сорбционные свойства титанцирконийсодержащего сорбента относительно извлечения урана из растворов типа морской воды.

В связи с этим с целью получения информации о влиянии механической обработки на разупорядоченность структуры и сорбционные характеристики сорбента проведены эксперименты по сорбции урана из моделирующих состав морской воды растворов с использованием измельченных образцов Термоксида-5.

\section{ЭКСПЕРИМЕНТАЛЬНАЯ ЧАСТЬ}

Эксперименты по изучению влияния механической обработки Термоксида-5 на его сорбционные свойства выполняли с использованием сорбента, содержащего 6.2 вес.\% $\mathrm{ZrO}_{2}$. Увеличение содержания $\mathrm{ZrO}_{2}$ выше 6.2 вес.\% не оказывает влияния на сорбцию урана, т.е. цирконий при более высоких концентрациях является инертной добавкой, не принимающей участия в процессе сорбции. Сорбент тщательно размалывали в агатовой ступке и методом седиментационного разделения отбирали фракции при времени осаждения $\leq 600$ с, затем высушивали при $22{ }^{\circ} \mathrm{C}$ и определяли гранулометрический состав. Характеристики сорбента с зернением $\sim 2.2 \cdot 10^{-3}$ см представлены в табл. 1 .

Эксперименты по сорбции урана приготовленными сорбентами проводили в статических услови- ях из модельного раствора морской воды с концентрацией урана $1.4 \cdot 10^{-4}$ моль/л и рН 7.85, в интервале температур 20-65 ${ }^{\circ} \mathrm{C}$ и времени от 0 до $3 \cdot 10^{4} \mathrm{c}$. Растворы готовили на основе морской воды Каспийского моря аналогично методике [10]. В воду вводили уран-233 и растворы природного урана, приготовленные растворением трикарбоната-уранилата натрия в морской воде.

Использование урана-233 в качестве радиоактивной метки обусловлено его $\alpha$-радиоактивностью $3.54 \cdot 10^{5}$ расп./с. мг, при этом суммарная удельная $\alpha$-радиоактивность природных изотопов урана пренебрежимо мала (25.8 расп./с. мг) и не влияет на результаты радиометрического определения урана-233.

Концентрацию урана в растворах определяли $\alpha$-радиометрически на приборе 2154-I-I М «ПРОTOKА».

Рентгено-дифрактограммы исследуемых образцов снимали на приборе «Дрон-2.0» с использованием $\mathrm{Cu}$ К $\alpha$-излучения с Ni-фильтром.

Кинетику сорбции урана на гидратированном сорбенте описывали уравнением Авраами-Ерофеева-Колмогорова[11]:

$$
\alpha_{\tau}=1-e^{-k \tau^{n}}
$$

где $\alpha_{\tau}-$ превращение фазы сорбента за время $\tau$, мольная доля; $k$ - константа скорости процесса сорбции, с; $n$ - постоянная величина, зависящая от условий эксперимента и механизма взаимодействия элемента с сорбентом.

Для расчёта величин $k$ и $n$ строили линеаризованные кинетические зависимости в координатах:

$$
\ln \left[-\ln \left(1-\frac{\alpha_{\tau}}{\alpha_{\infty}}\right)\right]-\ln \tau .
$$

Расчет величин $k$ и $n$ и их погрешностей производили с помощью метода наименьших квадратов. Так как величина $n$ в каждом случае непостоянна и из-за этого изменяется размерность величины

Таблица 1. Характеристики титан-цирконий содержащего сорбента

\begin{tabular}{|c|c|c|c|c|c|c|c|}
\hline \multicolumn{3}{|c|}{$\begin{array}{c}\text { Содерж., вес.\% } \\
\text { [Content, weight \%] }\end{array}$} & \multirow{2}{*}{$\begin{array}{c}S_{N_{2}}, \mathrm{M}^{2} / \Gamma \\
{\left[S_{N_{2}}, \mathrm{~m}^{2} / \mathrm{g}\right]}\end{array}$} & \multirow{2}{*}{$\begin{array}{c}\tau_{\text {эфф., }}, \mathrm{HM} \\
{\left[\tau_{\text {effect }}, \mathrm{nm}\right]}\end{array}$} & \multirow{2}{*}{$\begin{array}{c}V_{\text {уд }}, \mathrm{cm}^{3} / \Gamma \\
{\left[V_{\text {specific }}, \mathrm{cm}^{3} / \mathrm{g}\right]}\end{array}$} & \multirow{2}{*}{$\begin{array}{c}\rho_{\mathrm{H}}, \Gamma / \mathrm{cm}^{3} \\
{\left[\rho_{\mathrm{H}}, \mathrm{g} / \mathrm{cm}^{3}\right]}\end{array}$} & \multirow{2}{*}{$\begin{array}{l}P_{\mathrm{m}}, \mathrm{MПа} \\
P_{\mathrm{m}}, \mathrm{MPa}\end{array}$} \\
\hline $\mathrm{TiO}_{2}$ & $\mathrm{ZrO}_{2}$ & $\mathrm{H}_{2} \mathrm{O}$ & & & & & \\
\hline 77.3 & 6.2 & 16.5 & 270 & 1.8 & 0.319 & 1.01 & $36 \pm 6$ \\
\hline
\end{tabular}

[Table 1. Characteristics of titanium-zirconium sorbent which is used in the work]

$S_{N_{2}}$ - удельная поверхность, определенная по данным низкотемпературной адсорбции паров азота; $\tau_{\text {эфф. }}$ эффективный радиус пор; $\rho_{\text {н }}-$ насыпная плотность; $P_{\mathrm{m}}$ - механическая прочность гранул сорбента.

$S_{N_{2}}$ - specific surface, determined according to the data of low temperature adsorption of nitrogen vapor; $\tau_{\text {effect }}$ - effective pore radius; $\rho_{\mathrm{H}}$ - pour density; $P_{\mathrm{m}}$-mechanical density of sorbent granules. 
$k\left(\mathrm{c}^{-n}\right)$, то для удобства дальнейшей обработки и обсуждения экспериментов по сорбции рассчитывали обобщенные константы скоростей процесса сорбции урана $(K)$, имеющие постоянную размерность $\mathrm{c}^{-1}$, с помощью соотношения Саковича [12]:

$$
K=n \cdot R^{\frac{1}{n}} .
$$

\section{РЕЗУЛЬТАТЫ И ОБСУЖДЕНИЕ}

При изучении кинетических зависимостей сорбции, представленных на рис. 1, наблюдается ряд пиков, положение которых для каждой из температур определенно по времени. По мере выдерживания сорбента при комнатной температуре в течение трех суток или при его нагреве до $120^{\circ} \mathrm{C}$ в течение 1-1.5 часов пики уменьшаются и исчезают, а кинетические зависимости сорбции прини- мают традиционный вид (рис. 2). Это явление, не отмечавшееся ранее в литературе, позволяет предположить, что положение пиков и их воспроизводимость на кинетических зависимостях связаны с термическим отжигом свежеприготовленного порошка Термоксида-5. Получаемые равновесные коэффициенты распределения урана для свежеприготовленного и отожженного порошков, а также для исходного сорбента Термоксид-5 с размером частиц $1.0 \cdot 10^{-2} \mathrm{~cm}$ в пределах погрешности измерений равны между собой (табл. 2).

Для идентификации положения пиков методом наименьших квадратов производили обработку полученных кинетических зависимостей сорбции. Обработку проводили в тех областях зависимостей, где пики отсутствовали, после чего с по-

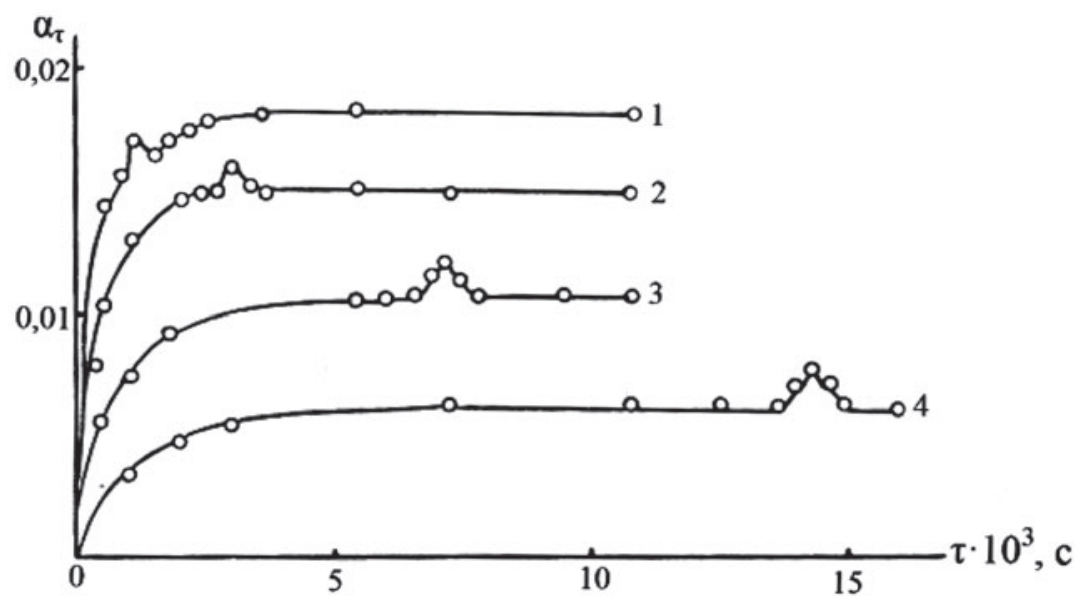

Рис. 1. Зависимости сорбции урана от времени свежеизмельченым Термоксидом-5 при различных температурах: 1 - 65.3; 2 - 50.2; 3 - 35.2; $4-20.1{ }^{\circ} \mathrm{C}$

[Fig. 1. Dependence of sorption of uranium on time with newly milled Thermoxide-5 at different temperatures: $1-65.3 ; 2-50.2 ; 3-35.2 ; 4-20.1^{\circ} \mathrm{C}$ ]

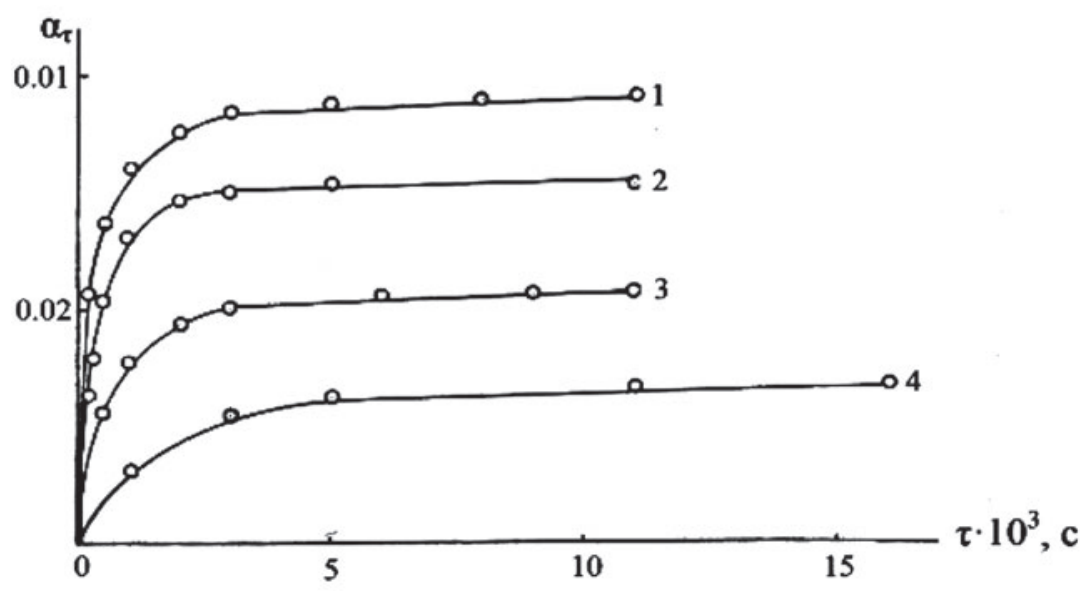

Рис. 2. Кинетические кривые сорбции урана измельченным Термоксидом-5 после его нагрева до $120^{\circ} \mathrm{C}$ в течение 1.5 часа при различных температурах: 1 - 65.3; 2 - 50.2; 3 - 35.2; 4 - $20.1{ }^{\circ} \mathrm{C}$

[Fig. 2. Dependence of sorption of uranium on time with newly milled Thermoxide- 5 after its heating till $120^{\circ} \mathrm{C}$ 1.5 hours at different temperatures: $\left.1-65.3 ; 2-50.2 ; 3-35.2 ; 4-20.1{ }^{\circ} \mathrm{C}\right]$ 
мощью вычисленных значений $\kappa$ и $n$ (табл. 3) рассчитывали значения $\alpha_{\tau}$ в областях пиков, затем эти значения вычитали из значений, определенных экспериментально.

Полученные величины разностей $\left(\Delta \alpha_{\tau}\right)$ откладывали в координатах $\Delta \alpha_{\tau}-\tau$, в результате получали рефлексы пиков, изображенные на рис. 3.

Обработка этих результатов методом наименьших квадратов в координатах $\ln \tau-1 / T$ выявила следующий вид зависимости:

$$
\ln \tau=-(6,4 \pm 2,8)-\frac{-(470 \pm 88)}{T}
$$

Если предположение о связи положения пиков на кинетических зависимостях сорбции с термическим отжигом дефектов решетки сорбента
Таблица. 2. Значение логарифмов коэффициентов распределения $\left(\ln K_{\mathrm{d}}\right)$ при сорбции урана из моделирующих состав морской воды растворов на Термоксиде-5

(6.2 вес.\% $\mathrm{ZrO}_{2}$ ) при температурах $293.2 \div 338.4 \mathrm{~K}$

[Table 2. Value of logarithms of distribution coefficients $\left(\ln K_{\mathrm{d}}\right.$ ) during sorption of uranium modeling compositions of seawater of solutionsThermoxide-5 (6.2 weight. $\% \mathrm{ZrO}_{2}$ ) and in the range of $293.2 \div 338.4 \mathrm{~K}$ ]

\begin{tabular}{|c|c|c|}
\hline \multirow{2}{*}{$\begin{array}{c}\text { Tемпература, K } \\
\text { [Temperature, K] }]\end{array}$} & \multicolumn{2}{|c|}{$\begin{array}{c}\text { Размер частиц сорбента, см } \\
\text { [Size of particles of a sorbent, sm] }\end{array}$} \\
\cline { 2 - 3 } & $1 \cdot 10^{-2}$ & $2.2 \cdot 10^{-3}$ \\
\hline 293.2 & 8.06 & 7.81 \\
\hline 308.3 & 8.05 & 8.17 \\
\hline 323.3 & 8.91 & 9.2 \\
\hline 338.4 & 9.97 & 9.60 \\
\hline
\end{tabular}

Таблица. 3. Результаты экспериментов по сорбции урана из моделирующих состав морской воды растворов на Термоксиде-5 с содержанием 6.2 вес.\% $\mathrm{ZrO}_{2}$, при $C_{\text {исх }}=1.4 \cdot 10^{-4}$ моль/л, $\mathrm{pH}=7.85$, с размером частиц $1.0 \cdot 10^{-2}$ см. Исходное мольное отношение урана к $\mathrm{TiO}_{2}$ в системе $\alpha_{0}=2.6 \cdot 10^{-2}$

[Table 3. Results of experiments on sorption of uranium from modeling compositions of seawater of solutions Thermoxide-5, with content of 6.2 weight $\% \mathrm{ZrO}_{2}$ at $C_{\text {primary }}=1.4 \cdot 10^{-4} \mathrm{~mol} / \mathrm{l}, \mathrm{pH}=7.85$, with size of particles $1.0 \cdot 10^{-2} \mathrm{sm}$, primary mole ratio of uranium to $\mathrm{TiO}_{2}$ in the system, $\alpha_{\infty}=2.6 \cdot 10^{-2}$ ]

\begin{tabular}{|c|c|c|c|c|c|c|}
\hline $\begin{array}{c}\text { Tемпература, K } \\
\text { [Temperature, K] }\end{array}$ & $\begin{array}{c}\text { Равновесное мольное } \\
\text { отношение урана К } \\
\mathrm{TiO}_{2} \text { в сорбенте, } \alpha_{\infty} \\
\text { [Equilibrium mole ratio } \\
\text { of uranium to } \mathrm{TiO}_{2} \text { in the } \\
\text { sorbent, } \alpha_{\infty} \text { ] }\end{array}$ & $\begin{array}{c}\text { Равновесная концент- } \\
\text { рация урана в растворе } \\
C_{\mathrm{p}, \boldsymbol{s}}, \text { моль/л } \\
\text { [Equilibrium concentra- } \\
\text { tion of uranium in } \\
\text { solution } C_{\mathrm{p}}, D \text {, mol//] }\end{array}$ & $\ln k$ & $n$ & $\ln K$ & $\ln K_{\mathrm{d}}$ \\
\hline 293.2 & $1.5 \cdot 10^{-2}$ & $5.9 \cdot 10^{-5}$ & -4.99 & 0.66 & -8.01 & 8.06 \\
\hline 308.3 & $1.5 \cdot 10^{-2}$ & $5.9 \cdot 10^{-5}$ & -4.81 & 0.67 & -7.62 & 8.05 \\
\hline 323.3 & $1.9 \cdot 10^{-2}$ & $3.3 \cdot 10^{-5}$ & -3.98 & 0.59 & -7.26 & 8.91 \\
\hline 338.4 & $2.3 \cdot 10^{-2}$ & $1.4 \cdot 10^{-5}$ & -3.45 & 0.52 & -7.29 & 9.97 \\
\hline
\end{tabular}

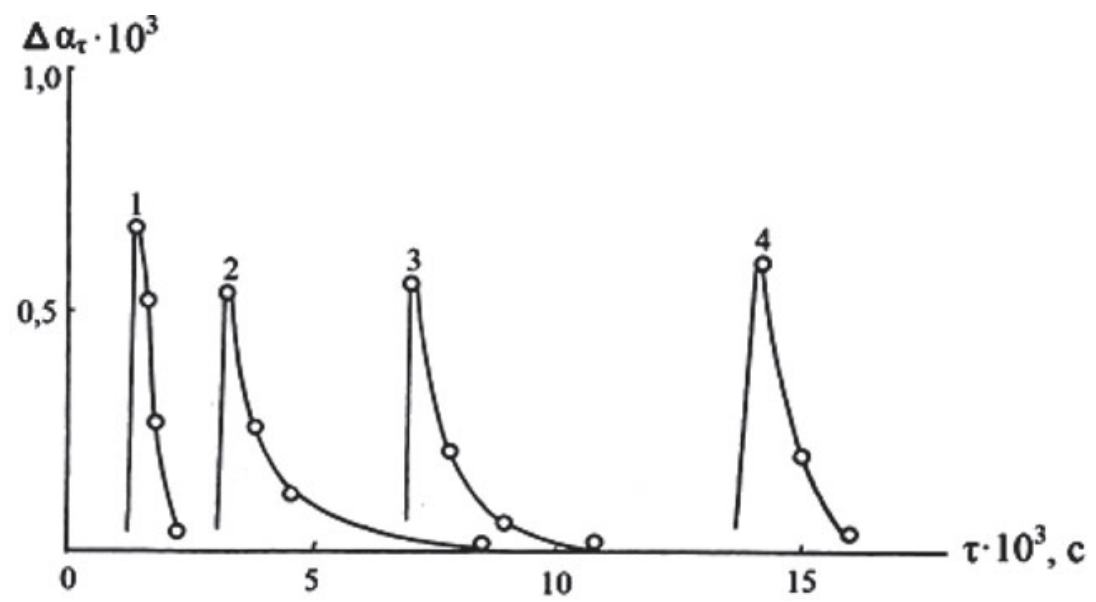

Рис. 3. Рефлексы пиков кинетических зависимостей сорбции, изображенных на рис. 1, при температурах сорбции: 1 - 65.3; 2 - 50.2; 3 - 35.2; 4 - $20.1^{\circ} \mathrm{C}$

[Fig. 3. Reflexes of peaks of kinetic dependences of sorption in fig. 1. $\left.1-65.3 ; 2-50.2 ; 3-35.2 ; 4-20.1^{\circ} \mathrm{C}\right]$ 
справедливо, то согласно уравнению Эйнштейна $[13,14]$ :

$$
\bar{X}^{2}=2 D \tau .
$$

где $\bar{X}^{2}$ - средняя величина квадрата расстояния, пройденного диффундирующим дефектом в данном направлении, см; $D$ - эффективный коэффициент диффузии дефектов, $\mathrm{cm}^{2} \cdot \mathrm{c}^{-1}$.

Полагая, что дефекты стекают (диффундируют) на наружную поверхность частиц сорбента [9], то есть $\bar{X}^{2}$ равен радиусу использованных частиц порошка $\left(\sim 5 \cdot 10^{-4} \mathrm{~cm}\right)$, можно записать, что

$$
2 D \tau=2.5 \cdot 10^{-7}
$$

Коэффициент диффузии в общем виде связан с температурой соотношением:

$$
D=D_{0} \cdot e^{-\frac{\Delta H}{R T}},
$$

где $D_{0}$ - предэкспоненциальный множитель, $\mathrm{cm}^{2} \cdot \mathrm{c}^{-1} ; \Delta H-$ энергия активации диффузии, Дж'моль ${ }^{-1} ; R-$ универсальная газовая постоянная, Дж·моль град ${ }^{-1}$.

Комбинируя уравнения (4), (6) и (7), получаем:

$$
D=7.83 \cdot 10^{-5} \cdot e^{-\frac{4703}{T}} .
$$

Согласно (8) энергия активации диффузии дефектов решетки Термоксида-5 равна 39.1 кДж·моль ${ }^{-1}$.

Рассчитанные на основании уравнения (8) эффективные коэффициенты диффузии дефектов в Термоксиде-5 при различных температурах представлены в табл. 4.

Очевидно, что наблюдаемые непосредственно после измельчения сорбционные пики связаны с полиморфным превращением в структуре сорбента. Сильная разупорядоченность кристаллической структуры исследованных сорбентов и их частичная аморфизация при интенсивном измельчении, возможно, сильно дестабилизируют структуру. В процессе дальнейшей термической стабилизации структуры происходит сток (диффузия) дефектов решетки и кристаллизация аморфных зон. Подобные процессы (полиморфные превращения) с перестройкой структуры обычно сопровождаются

Таблица. 4. Эффективные коэффициенты диффузии дефектов в Термоксиде-5 при различных температурах

[Table 4. Effective diffusion coefficients of defects in Thermoxide- 5 at different temperatures]

\begin{tabular}{|c|c|c|c|c|}
\hline$T,{ }^{\circ} \mathrm{C}$ & 20.1 & 35.2 & 50.2 & 65.3 \\
\hline $\begin{array}{c}D, \mathrm{~cm}^{2} \cdot \mathrm{c}^{-1} \\
D, \mathrm{~cm}^{2} \cdot \mathrm{c}^{-1}\end{array}$ & $8.5 \cdot 10^{-12}$ & $1.9 \cdot 10^{-11}$ & $3.8 \cdot 10^{-11}$ & $7.2 \cdot 10^{-11}$ \\
\hline
\end{tabular}

резким увеличением скоростей сорбции в момент структурного перехода (эффект Хедвалла [9]). Время наступления структурного перехода зависит от концентрации дефектов в твердой фазе, которая связана со скоростью их стока (диффузии) и различна для разных температур. Следовательно, полученные коэффициенты диффузии и энергия активации диффузионного процесса действительно отвечают коэффициентам самодиффузии и энергии активации диффузии материала сорбента.

С помощью рентгенофазового анализа был выявлен характер фазовых переходов в структуре сорбента, происходящих в результате измельчения и дальнейшей термической стабилизации. Для снятия дифрактограмм были приготовлены следующие образцы сорбента: исходный образец (зернение $1 \cdot 10^{-2} \mathrm{~cm}$ ), свежеизмельченный с размером частиц $2.2 \cdot 10^{-3}$ см и измельченный (размером $\sim 2.2 \cdot 10^{-3}$ см), выдержанный в течение трех суток до анализа при температуре $22{ }^{\circ} \mathrm{C}$.

На рис. 4 приведены дифрактограммы исследуемых образцов сорбента. Как следует из дифрактограммы (1), гидратированная двуокись титана в исходном сорбенте находится в основном в анатазной модификации с небольшой примесью рутила. Дифрактограмма (2) свежеизмельченного Термоксида-5 (зернение $2.2 \cdot 10^{-3} \mathrm{~cm}$ ) сильно размытая, со слабыми рефлексами указывает на значительную аморфизацию (разупорядоченность) исходной структуры сорбента. Дифрактограмма (3) выдержанного в течение трех суток при $22{ }^{\circ} \mathrm{C}$ измельченного образца идентична с дифрактограммой (1) исходного сорбента и имеет аналогичные рефлексы пиков. Это указывает на практически полное восстановление кристаллической структуры измельченного сорбента в результате выдержки при комнатной температуре в течение трех суток. Установлено аналогичное восстановление структуры измельченного образца при нагревании до $120{ }^{\circ} \mathrm{C}$ в течение $1-1.5$ часа.

Восстановление исходной анатазной структуры сорбента в результате измельчения с последующей выдержкой или отжигом указывает на то, что введение в ходе синтеза сорбента в гидратированную двуокись титана небольших количеств двуокиси циркония делает анатазную структуру сорбента термодинамически стабильной. Этот эффект связан, очевидно, с образованием ограниченного твердого раствора двуокиси циркония в двуокиси титана с верхним пределом смешиваемости I вес.\%. Этот факт обусловлен неизоморфностью кристаллических решеток двуокисей титана и циркония $\left(\mathrm{TiO}_{2}-\right.$ тетра- 


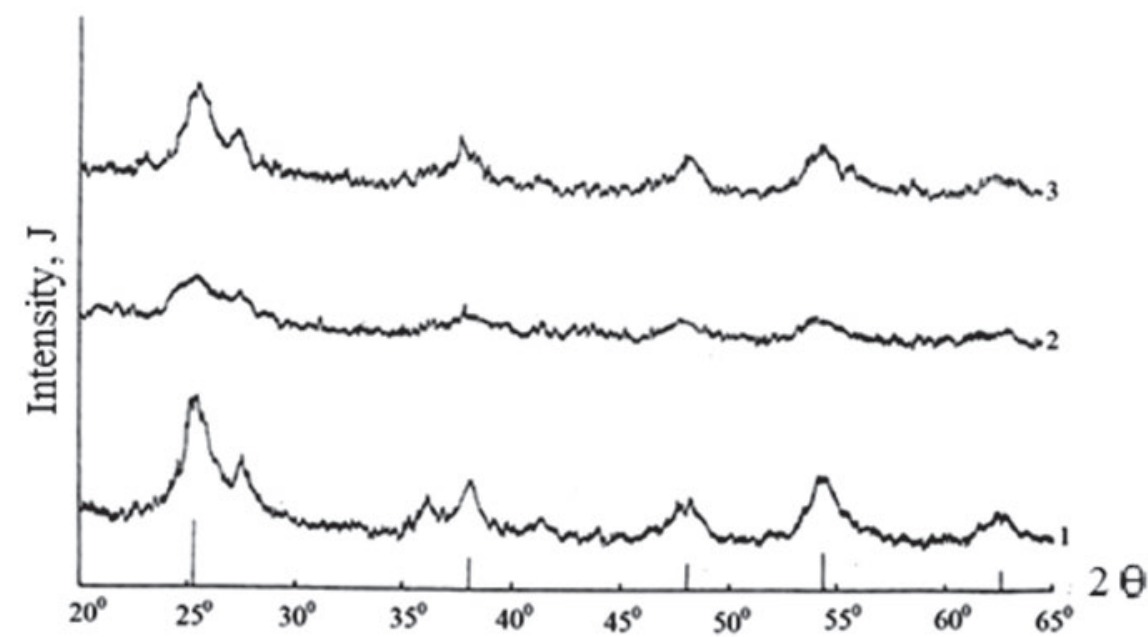

Рис. 4. Рентгено-дифрактограммы образцов сорбента термоксид-5 с содержанием $\mathrm{ZrO}_{2} 6.2$ вес.\%: 1 - исходный, с зернением $1 \cdot 10^{-2}$ см; 2 - свежеизмельченный с зернением $\sim 2 \cdot 10^{-3}$ см; $3-$ измельченный с зернением $\sim 2 \cdot 10^{-3}$ см, выдержанный в течение трех суток при $22{ }^{\circ} \mathrm{C}$. Внизу штрихрентгенограмма анатаза

[Fig. 4. X-ray-diffraction patterns of samples of Thermooxide-5 sorbent containing $\mathrm{ZrO}_{2} 6.2$ weight.\%: 1 - primary with granulation of $1 \cdot 10^{-2} \mathrm{sm} ; 2-$ newly milled with granulation of $\sim 2 \cdot 10^{-3} \mathrm{sm}$; $3-$ milled with granualation $\sim 2 \cdot 10^{-3} \mathrm{sm}$, stored 3 hours at $22{ }^{\circ} \mathrm{C}$. Below is streak X-ray pattern of anatase]

гональная решетка, $\mathrm{ZrO}_{2}$ - моноклинная решетка) вследствие разницы в значениях кристаллохимических радиусов ионов титана и циркония.

Результаты рентгенофазового анализа полностью подтверждают предположение о том, что наблюдаемое существенное изменение кинетики сорбции урана свежеизмельченным Термоксидом-5 (рис. 1) связано с аморфизацией (разупорядочением) структуры исходного сорбента. При выдержке или отжиге измельченного сорбента наблюдается полное восстановление сорбционных свойств (рис. 2), что связано с переходом аморфизированных форм сорбента в кристаллическую. При этом происходит стекание дефектов структуры сорбента и кристаллизация аморфных зон (рис. 4). Такого рода полиморфные превращения с перестройкой структуры в момент фазового перехода сопровождаются резким увеличением скоростей сорбции [9], что является причиной появления на кинетических зависимостях сорбции урана свежеизмельченным Термоксидом-5 характерных максимумов сорбции.

\section{ЗАКЛЮЧЕНИЕ}

Изучено влияние механической обработки сорбента Термоксид-5 с содержанием 6.2 вес. \% $\mathrm{ZrO}_{2}$ на его сорбционные свойства при извлечении урана из моделирующих состав морской воды растворов. При использовании свежеизмельченных мелкодисперсных фракций обнаружен эффект неравновесной разупорядоченности кристаллической решетки сорбента в виде пиков на кинетических кривых, исчезающих по истечению определенного времени.

Установлено, что причиной появления на кривых кинетических зависимостей сорбции урана свежеизмельченным Термоксидом-5 характерных максимумов сорбции является резкое увеличение скоростей сорбции, связанное с перестройкой структуры в момент фазового перехода, что подтверждено результатами рентгенофазового анализа.

Показано, что в процессе стабилизации структуры сорбента, происходящей при нагревании или выдержке его, происходит полное восстановление сорбционных свойств.

\section{СПИСОК ЛИТЕРАТУРЫ}

1. Шарыгин Л. М. Термостойкие неорганические сорбенты РАН. Уральское отделение, Институт химии твердого тела, Екатеринбург, 2012, с. 303.

2. Смирнова В. В. // Современные проблемы науки и образования, 2012, № 5, с. 1-7.

3. Комаревский В. М., Шарыгин Л. М., Гончар В. Ф., Новиков Ю. П., Малых Т. Г., Мясоедов Б. Ф. Сорбент на основе двуокиси титана для извлечения урана из солевых растворов. А.С. 899110 (СССР) от 21.09.81.

4. Мясоедов Б. Ф., Нуриев А. Н., Новиков Ю. П., Комаревский В. М., Мамедов Р. М., Шарыгин Л. М., Гончар В. Ф., Малых Т. Г. // Радиохимия, 1984, т. 26, № 3, c. $285-288$.

5. Нуриев А. Н., Акперов Г. А., Мамедов Р. М., Джаббарова 3. А., Рагимли М. А., Эфендиева Ш. 3. // Радиохимия, 1998, т. 40, с. 256-258. 
6. Нуриев А. Н., Рагимли М. А. // European Applied Science, 2013, № 7-2, pp. 97-101.

7. Рагимли М. А., Аббасов М. А., Эфендиева Ш. 3., Алиева В. Х., ИльясоваХ. А.«Успехи синтеза и комплексообразования», Тезисы докладов I Всероссийский молодежной школы-конференции, Москва, РУДН, 25-28 апреля, 2016, с. 252.

8. Смирнова В. В., Ильин А. П. // Фундаментальные исследования, 2013, № 6, ч. 6, с. 1366-1371.

9. Мейер К. Д. Физико-химическая кристаллография. М.: Металлургия, 1972, с. 480.
10. Рагимли М. А., Нуриев А. Н., Эфендиева Ш. З., Алиева В. Х., Ильясова Х. А. // Азерб. хим. журнал, 2011, №1, c. 23.

11. Еремин Е. М. Основы химической кинетики. М.: Высшая школа, 1976, с. 341.

12. Сакович Г. В. // Ученые записки Томского гос. университета, 1955, № 26, с. 103-110.

13. Heinicke V., Shober E. // Z. Chem., 1971, Bd. 11, № 6, pp. 219-226.

14. www.mbec.protres.ru/Programs/Serdyuk/2008/06. pp

\title{
THE INFLUENCE OF MECHANICAL PROCESSING ON THE STRUCTURE AND SORPTION PROPERTIES OF TITANIUM CONTAINING A SORBENT IN THE SORPTION OF URANIUM FROM SOLUTIONS MODELING COMPOSITIONS OF SEAWATER
}

\author{
(C) 2017 A. N. Nuriyev, M. A. Rahimli \\ Institute of Catalysis and Inorganic Chemistry named after acad.M. Nagiyevof ANAS, \\ 113 H. Javidave., AZE-1143 Baku, Azerbaijan \\ e-mail: chem@science.az
}

Received 26.04.2017

\begin{abstract}
The influence of mechanical processing of sorbent Thermoxide- 5 on its sorption properties, containing 6.2 weight $\% \mathrm{ZrO}_{2}$, during extraction of uranium from solutions modeling compositions of seawater.

The effect of the non-equilibrium disorder of the crystal lattice of the sorbent while using newly milled fine fractions (in the shape of peaks on kinetic curves) which disappear upon the expiration of a certain time was found. The values of activation energy and effective diffusion coefficients of defects in Thermoxide- 5 at different temperatures were calculated according to obtained results of the sorption kinetics.

It was established that the reason for the appearance of typical sorption peaks on kinetic dependences curves of uranium sorption by newly milled Thermoxide- 5 is sharp increase of sorption rates which is related to the rearrangement of structure in the moment of phase transition.

Based on XRD results it was determined that:

- Diffractgram of hydrated $\mathrm{TiO}_{2}$ in primary sorbent is, particularly, in anatase modification with small mixture of rutile.

- Diffractogram of a newly milled Thermoxide-5 (graining $~ 2.2 \cdot 10^{-3} \mathrm{sm}$ ) strongly blurred with weak reflexes points to the considerable amorphization (disorder) of primary structure of a sorbent.

- Diffractogram of a milled sample which was kept 3 days at $22^{\circ} \mathrm{C}$ temperature is identical to diffractogram of a primary sorbent and has similar reflexes of peaks, which points to practically full reduction of a crystalline structure of a milled sorbent which was kept at room temperature three days.

By this way, the study of sorption kinetics of uranium on newly milled and kept or heated (annealed) samples of a sorbent Thermoxide-5 showed that in sorption process of uranium from solutions with pH 7.85 thermodynamic equilibrium can be really achieved, but intensive grinding of sorbents leads to the destabilization of their structures. In the stabilization process of the structure of a sorbent during its heating or dwelling full reduction of sorption properties occurs. Consequently, disorder of the structure of a sorbent Thermoxide- 5 which is conditioned by the introduction of a isomorphic mixture of zirconium oxide to anatase lattice of hydrated titanium oxide, is thermally stable.
\end{abstract}

Keywords: uranium, titanium dioxide, sorbent, disorder of structure, sorption, XRD method. 


\section{REFERENCES}

1. Sharigin L. M. Heat-Proof Inorganic Sorbents RAS. Urals Branch Institute of Solid State Chemistry, Yekaterinburg Publ., 2012, p. 303. (in Russian)

2. Smirnova V. V. Modern Problems of Science and Education, 2012, no. 5, pp. 1-7.

3. Komarevskiy V. M., Sharigin L. M., Gonchar V. F., Novikov Y. P., Malikh T. G., Myasoyedov B. F. Sorbent on the Basis of Titanium Oxide for Extraction of Uranium From Saline Solution. A.S. 899110 (USSR) from 21.09.81.

4. Myasoyedov B. F., Nuriyev A. N., Novikov Y. P., Komarevskiy V. M., Mammadov R. M., Sharigin L. M., Gonchar V. F., Malikh T. G. Radiochemistry, 1984, vol. 26, no. 3, pp. 285-288.

5. Nuriyev A. N., Akperov G. A., Mammadov R. M., Jabbarova Z. A., Ragimli M. A., Efendiyeva Sh. Z. Radiochemistry, 1998, vol. 40, pp. 256-258.

6. Nuriyev A. N., Rahimli M. A. European Applied Science, 2013, no. 7-2, pp. 97-101.
7. Rahimli M. A., Abbasov M. A., Efenidyeva Sh. A., Aliyva V. Kh., Ilyasova Kh. A. "Advances in synthesis and complexing”, Theses of reports of I all-Russian conference for young scientists, Moscow, RUDN University, 2528 April, 2016, p. 252.

8. Smirnova V. V., Ilyin A. P. Fundamental researches, 2013, no. 6, ch. 6, pp. 1366-1371.

9. Meyer K. D. Physical-Chemical Crystallography. Moscow, Metallurgy Publ., 1972, p. 480. (in Russian)

10. Rahimli M. A., Nuriyev A. N., Efendiyeva Sh. Z., Aliyva V. Kh., Ilyasova Kh. A. Azerbaijan Chemistry Journal, 2011, no. 2, p. 23.

11. Eremin E. M. Fundamentals of Chemical Kinetics. Moscow, Higher School Publ., 1976, p. 341. (in Russian)

12. Sakovich G. V. Scientific Notes Tomsk State University,1955, no. 26, pp. 103-110.

13. Heinicke V., Shober E. Z. Chem., 1971, Bd.11, no. 6, pp. 219-226.

14. www.mbec.protres.ru/Programs/Serdyuk/2008/06. pp

Nuriev Ali Najafguluoglu - Correspondent member of ANAS, Professor, Head of Department, Head of Laboratory of Academician M. Nagiyev Institute of Catalysis and Inorganic Chemistry of ANAS; e-mail: chem@science.az

Rahimli Menzer Akhmed - Scientific Fellow of Academician M. Nagiyev Institute of Catalysis and Inorganic Chemistry of ANAS; e-mail: raqimova.seva@mail.ru 